\title{
Selective synthesis of functionalized, tertiary silanes by diastereoselective rearrangement-addition
}

\author{
Barry M. Trost, Zachary T. Ball, and Eun-Joo Kang
}

\author{
Supplemental Information
}

\section{(Z)-3-(Triethylsilyl)-dec-2-en-1-ol, 2}

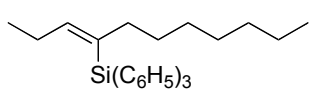

3-Decyn-1-ol (1.00 g, $6.48 \mathrm{mmol})$, and $\mathrm{Et}_{3} \mathrm{SiH}$ (1.23 mL, $\left.7.78 \mathrm{mmol}\right)$ were taken up in $\mathrm{CH}_{2} \mathrm{Cl}_{2}(13 \mathrm{~mL})$ under nitrogen and treated with solid $\left[\mathrm{Cp} * \mathrm{Ru}(\mathrm{MeCN})_{3}\right] \mathrm{PF}_{6}(33 \mathrm{mg}, 0.065 \mathrm{mmol})$ at $0{ }^{\circ} \mathrm{C}$. The solution was allowed to warm to rt and was stirred for $20 \mathrm{~min}$. The solvents were removed under reduced pressure, and the crude material was placed directly on a silica gel column for purification (eluent: 100:10:1 to 80:20:1 pet. ether: ether: methanol) to afford the product $(1.74 \mathrm{~g}, 99 \%)$ as a 13:1 mixture of regioisomers.

$\mathrm{R}_{\mathrm{f}}: 0.65$ (2:1 pet. ether: EtOAc). ${ }^{1} \mathrm{H}$ NMR $\left(300 \mathrm{MHz}, \mathrm{CDCl}_{3}\right) \delta 6.18(\mathrm{t}, J=7.1 \mathrm{~Hz}, 1 \mathrm{H}), 4.17(\mathrm{dd}, J=6.3,6.3 \mathrm{~Hz}$, 2H), $2.04(\mathrm{t}, J=6.3 \mathrm{~Hz}, 2 \mathrm{H}), 1.22-1.32(\mathrm{~m}, 13 \mathrm{H}), 1.15$ (t, $J=5.6 \mathrm{~Hz}, 1 \mathrm{H}), 0.86-0.97(\mathrm{~m}, 9 \mathrm{H}), 0.61-0.71(\mathrm{~m}, 6 \mathrm{H})$. ). ${ }^{13} \mathrm{C} \mathrm{NMR}\left(75 \mathrm{MHz}, \mathrm{CDCl}_{3}\right) \delta 141.4,141.0,62.3,38.1,31.8,30.5,29.5,29.2,22.6,14.1,7.5,4.3$. IR (thin film) 3317 (br), 2926, 2874, 1463, 1004, $732 \mathrm{~cm}^{-1}$. Anal. Calc. for $\mathrm{C}_{16} \mathrm{H}_{34} \mathrm{OSi}$ : C, 71.04; H, 12.67. Found: C, 71.21; $\mathrm{H}$, 12.76 .

\section{( \pm )-(2S,3R)-(3-Heptyl-3-triethylsilanyl-oxiranyl)-methanol 3}

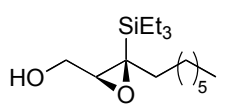

To a solution of vinylsilane 4.50 (400 mg, $1.48 \mathrm{mmol})$ in $\mathrm{CH}_{2} \mathrm{Cl}_{2}(20 \mathrm{~mL})$ was added $m$-CPBA (765 mg, 2.22 $\mathrm{mmol}, 50 \%$ ) at $0 \mathrm{oC}$. The white heterogeneous mixture was stirred for $1 \mathrm{~h}$. The reaction mixture was partitioned between $\mathrm{CH}_{2} \mathrm{Cl}_{2}$ and saturated aqueous sodium sulfite. The extract was washed with saturated aqueous $\mathrm{NaCl}$, dried

over anhydrous $\mathrm{MgSO}_{4}$, filtered, and the solvent was evaporated. The residue was purified by flash chromatography (eluent 6:1 to 4:1 pet. ether: ether) on silica gel to afford $430 \mathrm{mg}$ (quant.) of desired epoxy alcohol as a colorless oil.

$\mathrm{R}_{\mathrm{f}}: 0.52$ (4:1 pet. ether: EtOAc). ${ }^{1} \mathrm{H}$ NMR $\left(300 \mathrm{MHz}, \mathrm{CDCl}_{3}\right)_{-} 3.84\left(\mathrm{ABX}, J_{\mathrm{AB}}=12.0, J_{\mathrm{AX}}=3.0 \mathrm{~Hz}, 1 \mathrm{H}\right), 3.56$ $\left(\mathrm{ABX}, J_{\mathrm{AB}}=12.0, J_{\mathrm{BX}}=8.1 \mathrm{~Hz}, 1 \mathrm{H}\right), 2.96(\mathrm{dd}, J=8.1,3.0 \mathrm{~Hz}, 1 \mathrm{H}), 1.81-1.94(\mathrm{~m}, 1 \mathrm{H}), 1.80(\mathrm{br}, \mathrm{O}-\mathrm{H}), 1.06-1.22$ $(\mathrm{m}, 11 \mathrm{H}), 0.97(\mathrm{t}, J=8.0 \mathrm{~Hz}, 9 \mathrm{H}), 0.86(\mathrm{t}, J=6.6 \mathrm{~Hz}, 3 \mathrm{H}), 0.56-0.69(\mathrm{~m}, 6 \mathrm{H}) .{ }^{13} \mathrm{C} \mathrm{NMR}\left(75 \mathrm{MHz}, \mathrm{CDCl}_{3}\right) \_63.3$, 63.2, 57.2, 37.4, 31.7, 29.9, 29.1, 25.4, 22.6, 14.0, 7.5, 3.4. IR (thin film) 3418 (br. O-H), 3056, 2930, 2876, 1465, 1006, $732 \mathrm{~cm}^{-1}$. HRMS_EI (m/z): [M] calcd for $\mathrm{C}_{16} \mathrm{H}_{34} \mathrm{O}_{2} \mathrm{Si}: 286.2328$; found: 286.2323 .

\section{( \pm )-(2S,3S)-2-Heptyl-2-triethylsilyl-1,3-butanediol, 6a}




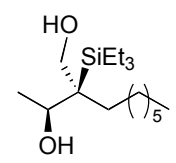

Note: The silyl diol rearrangement products are quite unstable, especially to mild acid, and NMR analysis of these compounds is best performed in benzene- $d 6$, to avoid traces of acid impurities present in $\mathrm{CDCl}_{3}$.

To a stirred solution of $\mathrm{CuCN}(90 \mathrm{mg}, 1.0 \mathrm{mmol})$ in ether $(1.5 \mathrm{~mL})$ under $\mathrm{Ar}$ at $-45 \mathrm{oC}$ was added a $3 \mathrm{M}$ solution of methylmagnesium bromide in $\mathrm{Et}_{2} \mathrm{O}(0.7 \mathrm{~mL})$ dropwise. The epoxy alcohol 3 in ether $(1 \mathrm{~mL})$ was added slowly via cannula. The heterogeneous mixture was stirred and warmed slowly to r.t. After $6 \mathrm{~h}$, the reaction was quenched with $90 \% \mathrm{NH} 4 \mathrm{Cl}: 10 \% \mathrm{NH} 4 \mathrm{OH}$ solution. After stirring at r.t. for ca. $30 \mathrm{~min}$, the solution was transferred to a separatory funnel, and $5 \mathrm{~mL}$ of a saturated aqueous $\mathrm{NaCl}$ solution was added. This was then extracted with ether, and the extracts were dried over $\mathrm{MgSO}_{4}$. Following filtration, the ether was removed in vacuo, and the residue was chromatographed on silica gel (eluent 3:1 pet. ether:ether) to afford $36 \mathrm{mg}(68 \%)$ of the diol with recovered starting material. (6 mg, $11 \%)$.

Rf: 0.43 (4:1 pet. ether:EtOAc). ${ }^{1} \mathrm{H}$ NMR $\left(300 \mathrm{MHz}, \mathrm{C}_{6} \mathrm{D}_{6}\right) \_4.12(\mathrm{q}, J=6.2 \mathrm{~Hz}, 1 \mathrm{H}), 3.77$ and 3.85 (AB, $J=10.5$ $\mathrm{Hz}, 2 \mathrm{H}), 3.27(\mathrm{br}, 2 \mathrm{H}), 1.65-1.80(\mathrm{~m}, 1 \mathrm{H}), 1.43-1.58(\mathrm{~m}, 1 \mathrm{H}), 1.22-1.38(\mathrm{~m}, 10 \mathrm{H}), 1.17(\mathrm{~d}, J=6.3 \mathrm{~Hz}, 3 \mathrm{H}), 1.05(\mathrm{t}$, $J=7.9 \mathrm{~Hz}, 9 \mathrm{H}), 0.91(\mathrm{t}, J=6.6 \mathrm{~Hz}, 3 \mathrm{H}), 0.73(\mathrm{q}, J=7.8 \mathrm{~Hz}, 6 \mathrm{H}) .{ }^{13} \mathrm{C} \mathrm{NMR}\left(75 \mathrm{MHz}, \mathrm{C}_{6} \mathrm{D}_{6}\right) 72.9,67.5,37.6,32.4$, 31.5, 29.7, 29.0, 26.1, 23.1, 19.36, 14.3, 8.6, 4.3. Anal. calc. for $\mathrm{C}_{17} \mathrm{H}_{38} \mathrm{O}_{2} \mathrm{Si}: \mathrm{C}, 67.48 ; \mathrm{H}, 12.66$; Found: C, 68.77; H, 12.82 .

\section{( \pm )-(2S,3S)-2-(Benzyldimethylsilyl)-2-heptyl-butane-1,3-diol, 6b}

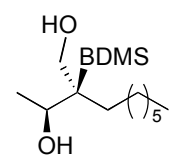

Neat $( \pm)-(2 S, 3 R)-(3-H e p t y l-3$-benzyldimethylsilyl-oxiranyl)-methanol (54 mg, $0.17 \mathrm{mmol})$ and $2.5 \mathrm{M}$ solution of $\mathrm{MeMgBr}$ in ether $(0.2 \mathrm{~mL})$ were treated as in the preparation of diol 11 to give the title compound (35 $\mathrm{mg}, 61 \%$ ) after the silica gel chromatography (eluent 2:1 pet. ether: ether).

$\mathrm{R}_{\mathrm{f}}$ : 0.30 (4:1 pet. ether:EtOAc). ${ }^{1} \mathrm{H}$ NMR $\left(300 \mathrm{MHz}, \mathrm{C}_{6} \mathrm{D}_{6}\right)$ _ 7.16-7.20 (m, 2H), 7.02-7.08 (m, 3H), 4.00 (q, $J=6.1$ $\mathrm{Hz}, 1 \mathrm{H}), 3.53$ and $3.74\left(\mathrm{AB}, J_{\mathrm{AB}}=10.4 \mathrm{~Hz}, 2 \mathrm{H}\right), 2.32(\mathrm{~s}, 2 \mathrm{H}), 2.06$ (br, $\left.1 \mathrm{H}\right)$, Rf: 0.45 (4:1 pet. ether:EtOAc). $1 \mathrm{H}$ $\operatorname{NMR}\left(300 \mathrm{MHz}, \mathrm{CDCl}_{3}\right) \_7.21-7.34(\mathrm{~m}, 5 \mathrm{H}), 4.98(\mathrm{~s}, 1 \mathrm{H}), 3.60-3.68(\mathrm{~m}, 2 \mathrm{H}), 2.95(\mathrm{br}, 1 \mathrm{H}), 2.61(\mathrm{br}, 1 \mathrm{H}), 1.08-$ $1.37(\mathrm{~m}, 12 \mathrm{H}), 1.08-1.37(\mathrm{~m}, 12 \mathrm{H}), 1.04(\mathrm{t}, J=7.8 \mathrm{~Hz}, 9 \mathrm{H}), 0.86(\mathrm{t}, J=6.8 \mathrm{~Hz}, 3 \mathrm{H}), 0.79(\mathrm{q}, J=7.4 \mathrm{~Hz}, 6 \mathrm{H}) .{ }^{13} \mathrm{C}$ NMR (75 MHz, $\left.\mathrm{C}_{6} \mathrm{D}_{6}\right) \_$141.69, 128.0, 127.5, 127.4, 79.1, 66.7, 38.2, 31.8, 31.7, 30.7, 29.1, 25.4, 22.6, 14.1, 8.5, 4.2. IR (thin film) 3333, 2954, 2928, 2875, 1454, 1059, 1010, 722, $702 \mathrm{~cm}^{-1}$. HRMS-EI (m/z): $\left[\mathrm{M}-\mathrm{H}_{2} \mathrm{O}\right]^{+}$calcd for $\mathrm{C}_{20} \mathrm{H}_{34} \mathrm{OSi}$ : 318.2379 ; found: 318.2374 .

\section{(E)-2-Ethylidene-1-nonanol, 10}

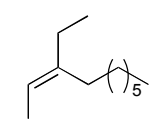


The reaction solution of the diol $6 \mathbf{a}(33.7 \mathrm{mg}, 0.11 \mathrm{mmol})$ in ether $(2.5 \mathrm{~mL})$ was cooled to $-78^{\circ} \mathrm{C}$. TFA ( 3 drops, catalytic amount) was added, and the solution was warmed over $2 \mathrm{~h}$ to r.t. and stirred for $1 \mathrm{~h}$. The reaction mixture was diluted with ether, washed with saturated aqueous $\mathrm{NaHCO}_{3}$, and $\mathrm{NaCl}$ solution, dried over $\mathrm{MgSO}_{4}$, and the solvent was evaporated. The purification by silica gel chromatography (eluent 6:1 pet. ether:ether) afforded the alcohol (14 mg, $75 \%)$.

Rf: 0.72 (4:1 pet. ether:EtOAc). ${ }^{1} \mathrm{H}$ NMR $\left(500 \mathrm{MHz}, \mathrm{CDCl}_{3}\right) \_5.50$ (q, $\left.J=4.0 \mathrm{~Hz}, 1 \mathrm{H}\right), 4.03(\mathrm{~s}, 2 \mathrm{H}), 2.10$ (t, $J=4.7$ $\mathrm{Hz}, 2 \mathrm{H}), 1.64(\mathrm{~d}, J=4.2 \mathrm{~Hz}, 3 \mathrm{H}), 1.18-1.41(\mathrm{~m}, 11 \mathrm{H}), 0.89(\mathrm{t}, J=4.1 \mathrm{~Hz}, 3 \mathrm{H}) .{ }^{13} \mathrm{C} \mathrm{NMR}\left(75 \mathrm{MHz}, \mathrm{CDCl}_{3}\right)_{-} 140.1$, 121.0, 67.3, 31.9, 29.7, 29.2, 28.3, 27.7, 22.7, 14.1, 13.0. IR (thin film) 3330, 2949, 2925, 2856, 1623, 1458, 1071, $1008 \mathrm{~cm}^{-1}$. HRMS—EI (m/z): [M] calcd for $\mathrm{C}_{11} \mathrm{H}_{22} \mathrm{O}: 170.1671$; found: 170.1677 .

\section{General procedure for the addition of Grignard reagents to $\beta$-silyl- $\alpha, \beta$-epoxy alcohols: $( \pm)-(1 S, 2 R)-2-H e p t y l-$} 1-phenyl-2-triethylsilyl-1,3-propanediol, 11

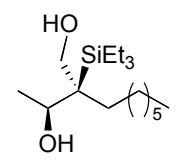

To a $25 \mathrm{~mL}$ round-bottom flask charged with $1.5 \mathrm{~mL}$ of dry ether, the $2.44 \mathrm{M}$ solution of $\operatorname{PhMgBr}(0.22 \mathrm{~mL})$ in ether was added at $-20^{\circ} \mathrm{C}$, and then the epoxy alcohol $11(50 \mathrm{mg}, 0.17 \mathrm{mmol})$ in ether $(1 \mathrm{~mL})$ was added via cannula. The reaction mixture was warmed slowly to r.t. for $6 \mathrm{~h}$, and partitioned between ether and saturated aqueous $\mathrm{NH}_{4} \mathrm{Cl}$ solution. The ethereal extract was washed with saturated aqueous $\mathrm{NaCl}$ solution, dried over $\mathrm{MgSO}_{4}$, and filtered, and the the solvent was evaporated. The residue was flash chromatographed on silica gel (eluent 3:1 pet. ether:ether) to afford $41 \mathrm{mg}$ ( $65 \%)$ of the destitle compound as a colorless oil.

Rf: 0.45 (4:1 pet. ether: EtOAc). ${ }^{1} \mathrm{H}$ NMR (300 MHz, $\left.\mathrm{CDCl}_{3}\right)_{-}$7.21-7.34 (m, 5H), $4.98(\mathrm{~s}, 1 \mathrm{H}), 3.60-3.68(\mathrm{~m}, 2 \mathrm{H})$, 2.95 (br, 1H), $2.61(\mathrm{br}, 1 \mathrm{H}), 1.08-1.37(\mathrm{~m}, 12 \mathrm{H}), 1.08-1.37(\mathrm{~m}, 12 \mathrm{H}), 1.04(\mathrm{t}, J=7.8 \mathrm{~Hz}, 9 \mathrm{H}), 0.86(\mathrm{t}, J=6.8 \mathrm{~Hz}$, $3 \mathrm{H}), 0.79$ (q, $J=7.4 \mathrm{~Hz}, 6 \mathrm{H}) .{ }^{13} \mathrm{C} \mathrm{NMR}\left(75 \mathrm{MHz}, \mathrm{CDCl}_{3}\right)_{-} 141.69,128.0,127.5,127.4,79.1,66.7,38.2,31.8$, $31.7,30.7,29.1,25.4,22.6,14.1,8.5,4.2$. IR (thin film) 3333, 2954, 2928, 2875, 1454, 1059, 1010, 722, $702 \mathrm{~cm}^{-1}$

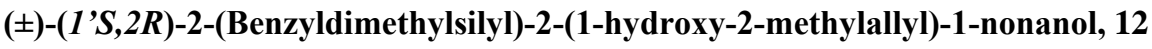

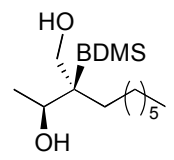

The epoxy alcohol $(64 \mathrm{mg}, 0.20 \mathrm{mmol})$ and $0.4 \mathrm{M}$ solution of isopropenylmagnesium bromide in THF (1.0 $\mathrm{mL}$ ) with ether $(2.5 \mathrm{~mL})$ as a solvent were treated as in the preparation of diol $\mathbf{1 1}$ to give the desired product (61 mg, $86 \%$ ) after the silica gel chromatography (eluent 6:1 pet. ether:ether).

$\mathrm{R}_{\mathrm{f}}$ : 0.65 (4:1 pet. ether: EtOAc). ${ }^{1} \mathrm{H}$ NMR (400 MHz, $\left.\mathrm{CDCl}_{3}\right)$ _ 7.19-7.24 (m, 2H), 7.03-7.10 (m, 3H), 5.02-5.04 (m, $2 \mathrm{H}), 4.47(\mathrm{~d}, J=10.8 \mathrm{~Hz}, 1 \mathrm{H}), 3.86$ and $3.72(\mathrm{ABX}, J \mathrm{AB}=10.8, J \mathrm{AX}=3.2, J \mathrm{BX}=4.4 \mathrm{~Hz}, 2 \mathrm{H}), 2.42(\mathrm{br}, 1 \mathrm{H}), 2.36$ $(\mathrm{d}, J=4.0 \mathrm{~Hz}, 1 \mathrm{H}), 2.34$ (s, 2H), 1.87 (s, 3H), 1.33-1.43 (m, 3H), 1.19-1.31 (m, 9H), 0.89 (t, $J=7.0 \mathrm{~Hz}, 3 \mathrm{H}), 0.06$ (s, 3H), 0.05 (s, 3H). ${ }^{13} \mathrm{C}$ NMR (75 MHz, $\left.\mathrm{CDCl}_{3}\right) \_$147.0, 140.7, 128.5, 128.4, 128.0, 123.9, 113.9, 78.9, 67.0, 37.7, 
32.0, 31.9, 30.8, 29.1, 25.4, 25.2, 22.6, 22.1, 14.1, -2.9, -2.9. IR (thin film) 3492, 2956, 2928, 2855, 1744, 1728, 1452, 1368, 1248, 1032, 912, 827, $700 \mathrm{~cm}^{-1}$. Anal. calc. for $\mathrm{C}_{22} \mathrm{H}_{38} \mathrm{O}_{2} \mathrm{Si}: \mathrm{C}, 72.87 ; \mathrm{H}, 10.56$; Found: C, 73.09; $\mathrm{H}$, 10.36 .

\section{$( \pm)-(2 R, 3 S)-2-H e p t y l-2-t r i e t h y l s i l y l-4-p e n t y n e-1,3-d i o l, 6.146 a$ and 1,4-enyne, 13a and 13b}

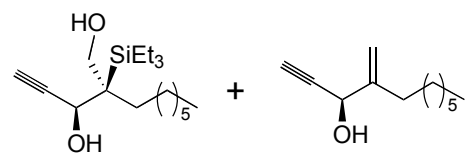

The epoxy alcohol $\mathbf{3 b}(55 \mathrm{mg}, 0.19 \mathrm{mmol})$ and $0.5 \mathrm{M}$ solution of ethynylmagnesium bromide in THF (0.96 $\mathrm{mL})$ were treated as in the preparation of diol 11 to give the desired product 13a $(34 \mathrm{mg}, 58 \%)$ and the elimination product $\mathbf{1 3 b}(6.5 \mathrm{mg}, 18 \%)$ after the silica gel chromatography (eluent: 4:1 pet. ether: ether).

Data for diol 13a:

Rf: 0.48 (4:1 pet. ether: EtOAc). ${ }^{1} \mathrm{H}$ NMR $\left(300 \mathrm{MHz}, \mathrm{CDCl}_{3}\right)_{-} 4.65$ (br m, 1H), 3.87 (br d, $\left.2 \mathrm{H}\right), 3.16$ (d, J=6.0 Hz, $1 \mathrm{H}), 2.54(\mathrm{~d}, J=2.1 \mathrm{~Hz}, 1 \mathrm{H}), 2.28(\mathrm{br}, 1 \mathrm{H}), 1.19-1.46(\mathrm{~m}, 12 \mathrm{H}), 0.93-1.01(\mathrm{~m}, 9 \mathrm{H}), 0.86(\mathrm{t}, J=6.6 \mathrm{~Hz}, 3 \mathrm{H}), 0.69-$

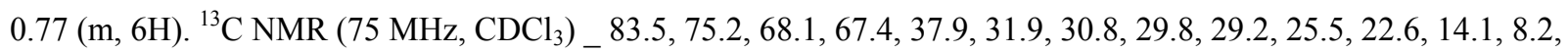
3.7. IR (thin film) $3310,2955,2928,2876,1466,1419,1242,1010,723 \mathrm{~cm}^{-1}$. Anal. calc. for $\mathrm{C}_{18} \mathrm{H}_{36} \mathrm{O}_{2} \mathrm{Si}: \mathrm{C}, 69.17$; H, 11.61; Found: C, 69.09; H, 11.74.

Data for 1,4-enyne 13b:

Rf: 0.60 (4:1 pet. ether:EtOAc). ${ }^{1} \mathrm{H}$ NMR $\left(400 \mathrm{MHz}, \mathrm{CDCl}_{3}\right) \_5.30(\mathrm{t}, J=1.0 \mathrm{~Hz}, 1 \mathrm{H}), 4.97(\mathrm{t}, J=0.6 \mathrm{~Hz}, 1 \mathrm{H})$, 4.82 (br s, 1H), 2.55 (d, $J=2.4 \mathrm{~Hz}, 1 \mathrm{H}), 2.20$ (t, $J=7.8 \mathrm{~Hz}, 2 \mathrm{H}), 1.91$ (br, 1H), 1.46-1.52 (m, 2H), 1.21-1.31 (m, $10 \mathrm{H}), 0.88(\mathrm{t}, J=7.0 \mathrm{~Hz}, 3 \mathrm{H}) .{ }^{13} \mathrm{C} \mathrm{NMR}\left(100 \mathrm{MHz} \mathrm{CDCl}_{3}\right)_{-}$147.8, 111.4, 83.0, 74.1, 65.4, 31.8, 31.7, 29.4, 29.2, 27.7, 22.6, 14.1. IR (thin film) 3312, 2956, 2927, 2856, 1465, 1378, 1261, 1021, 908, 722, $628 \mathrm{~cm}^{-1}$. Anal. calc. for $\mathrm{C}_{18} \mathrm{H}_{36} \mathrm{O}_{2} \mathrm{Si}: \mathrm{C}, 69.17$; H, 11.61; Found: C, 69.09; H, 11.74.

\section{( \pm )-(2S,3R)-3-Allyl-3-(benzyldimethylsilyl)-1,2-decanediol, 14}

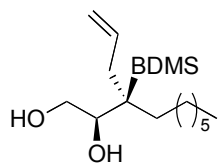

The epoxy alcohol $(68 \mathrm{mg}, 0.21 \mathrm{mmol})$ and $0.5 \mathrm{M}$ solution of allylmagnesium bromide in ether $(1.12 \mathrm{~mL})$ were treated as in the preparation of diol 11 to give the desired product $(65 \mathrm{mg}, 84 \%)$ after the silica gel chromatography (eluent 3:1 pet. ether:ether).

Rf: 0.36 (4:1 pet. ether:EtOAc). ${ }^{1} \mathrm{H}$ NMR $\left(400 \mathrm{MHz}, \mathrm{CDCl}_{3}\right)$ _ $7.21(\mathrm{t}, J=7.6 \mathrm{~Hz}, 2 \mathrm{H}), 7.07$ (t, $\left.J=7.2 \mathrm{~Hz}, 1 \mathrm{H}\right), 7.00$ $(\mathrm{d}, J=7.2 \mathrm{~Hz}, 2 \mathrm{H}), 5.93(\mathrm{ddt}, J=17.2,10.0,7.2 \mathrm{~Hz}, 1 \mathrm{H}), 5.15(\mathrm{dd}, J=17.2,2.2 \mathrm{~Hz}, 1 \mathrm{H}), 5.10(\mathrm{dd}, J=10.0,2.2 \mathrm{~Hz}$, $1 \mathrm{H}), 3.93(\mathrm{dd}, J=9.2,2.4 \mathrm{~Hz}, 1 \mathrm{H}), 3.63-3.74(\mathrm{~m}, 2 \mathrm{H}), 2.49$ (dd, $J=14.0,7.6 \mathrm{~Hz}, 1 \mathrm{H}), 2.33$ (br, $1 \mathrm{H}), 2.24$ and 2.26 $\left(\mathrm{ABq}, J_{\mathrm{AB}}=13.6 \mathrm{~Hz}, 2 \mathrm{H}\right), 2.15(\mathrm{dd}, J=14.0,7.6 \mathrm{~Hz}, 1 \mathrm{H}), 1.96(\mathrm{br}, 1 \mathrm{H}), 1.60-1.67(\mathrm{~m}, 1 \mathrm{H}), 1.21-1.33(\mathrm{~m}, 11 \mathrm{H})$, $0.89(\mathrm{t}, J=7.0 \mathrm{~Hz}, 3 \mathrm{H}), 0.01(\mathrm{~s}, 3 \mathrm{H}), 0.00(\mathrm{~s}, 3 \mathrm{H}) .{ }^{13} \mathrm{C} \mathrm{NMR}\left(75 \mathrm{MHz}, \mathrm{CDCl}_{3}\right)_{\text {_ }}$ 140.2, 136.4, 128.5, 128.1, 124.0, $117.5,75.7,63.4,37.5,33.1,32.9,31.9,30.9,29.2,25.2,22.6,14.1,-2.6$. IR (thin film) 3388, 2929, 2855, 1600, 
1493, 1452, 1251, 1204, 1059, 1030, 826, $699 \mathrm{~cm}^{-1}$. Anal. calc. for $\mathrm{C}_{22} \mathrm{H}_{38} \mathrm{O}_{2} \mathrm{Si}: \mathrm{C}, 72.87$; H, 10.56; Found: C, $73.02 ; \mathrm{H}, 10.42$.

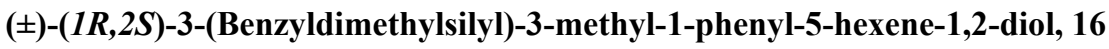

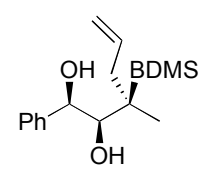

Epoxy alcohol $15(34 \mathrm{mg}, 0.11 \mathrm{mmol})$ and $0.5 \mathrm{M}$ solution of allylmagnesium bromide in ether $(0.65 \mathrm{~mL})$ were treated as in the preparation of diol 11 to give the desired product (34 $\mathrm{mg}, 88 \%$ ) after the silica gel chromatography (eluent: 10:1 pet. ether: ether).

Rf: 0.80 (4:1 pet. ether: EtOAc). ${ }^{1} \mathrm{H}$ NMR $\left(500 \mathrm{MHz}, \mathrm{CDCl}_{3}\right){ }_{-}$7.29-7.42 (m, 4H), $7.21(\mathrm{t}, J=7.5 \mathrm{~Hz}, 2 \mathrm{H}), 7.08(\mathrm{t}, J$ $=7.5 \mathrm{~Hz}, 1 \mathrm{H}), 7.03(\mathrm{~d}, J=7.0 \mathrm{~Hz}, 2 \mathrm{H}), 6.03(\mathrm{ddt}, J=17.0,10.0,7.5 \mathrm{~Hz}, 1 \mathrm{H}), 5.20(\mathrm{dd}, J=17.0,1.5 \mathrm{~Hz}, 1 \mathrm{H}), 5.14$ $(\mathrm{dd}, J=10.0,1.0 \mathrm{~Hz}, 1 \mathrm{H}), 4.92(\mathrm{dd}, J=6.0,1.5 \mathrm{~Hz}, 1 \mathrm{H}), 3.72(\mathrm{dd}, J=6.7,1.7 \mathrm{~Hz}, 1 \mathrm{H}), 2.63(\mathrm{~d}, J=6.0 \mathrm{~Hz}, 1 \mathrm{H})$, $2.60(\mathrm{~d}, J=6.5 \mathrm{~Hz}, 1 \mathrm{H}), 2.55(\mathrm{dd}, J=14.2,7.3 \mathrm{~Hz}, 1 \mathrm{H}), 2.36(\mathrm{dd}, J=14.0,7.5 \mathrm{~Hz}, 1 \mathrm{H}), 2.27$ and $2.32(\mathrm{ABq}, J \mathrm{AB}=$ $13.5 \mathrm{~Hz}, 2 \mathrm{H}), 1.04(\mathrm{~s}, 3 \mathrm{H}), 0.04(\mathrm{~s}, 3 \mathrm{H}), 0.01(\mathrm{~s}, 3 \mathrm{H}) .{ }^{13} \mathrm{C} \mathrm{NMR}\left(75 \mathrm{MHz}, \mathrm{CDCl}_{3}\right)_{-}$143.9, 140.5, 136.6, 128.6, 128.4, 128.0, 127.6, 125.8, 123.9, 117.2, 80.3, 70.9, 38.1, 31.0, 24.4, 18.8, -3.8. IR (thin film) 3538, 3387, 2926, $1493,1452,1401,1247,1060,913,827,700 \mathrm{~cm}^{-1}$. 